\title{
The association between melatonin receptor 1B gene polymorphisms and type 2 diabetes mellitus (T2DM) in Chinese populations: a meta-analysis
}

\author{
Xinxia Zhang, Lanlan Xie, Min Zhong, Botong Yang, Qiyue Yang, Hui Yang, Chunguang Xie \\ Department of Endocrinology, Hospital of Chengdu University of Traditional Chinese Medicine, Chengdu 610072, China \\ Contributions: (I) Conception and design: X Zhang, C Xie; (II) Administrative support: X Zhang, L Xie, B Yang; (III) Provision of study materials: L \\ Xie, M Zhong, Q Yang, H Yang; (IV) Collection and assembly of data: X Zhang, M Zhong, Q Yang; (V) Data analysis and interpretation: X Zhang, L \\ Xie, H Yang, C Xie; (VI) Manuscript writing: All authors; (VII) Final approval of manuscript: All author \\ Correspondence to: Chunguang Xie. Department of Endocrinology, Hospital of Chengdu University of Traditional Chinese Medicine, No. 39, Shi Er \\ Qiao Road, Jinniu District, Chengdu 610072, China. Email: xiechunguangcg@163.com.
}

Background: Type 2 diabetes mellitus (T2DM) is associated with a large number of genetic variants of melatonin receptor 1B (MTNR1B), but the results of studies involving different racial groups have been inconsistent. Thus, we carried out a meta-analysis to evaluate the correlation between the MTNR1B variants and T2DM in the Chinese population.

Methods: A systematic search was conducted of English-language databases including PubMed, Embase, and Medline, and Chinese-language databases including China National Knowledge Infrastructure (CNKI), Wanfang Database, and CQVIP to collect relevant articles published up to January 31, 2020. Count data were pooled using odds ratio (OR) and $95 \%$ confidence interval (CI), and the analysis was performed using the "meta" package of the R3.5.1 software.

Results: Nine articles involving 10,127 subjects in the T2DM group and 10,885 subjects in the healthy control group were entered into the final analysis. Four articles reported the rs1387153 variant of the MTNR1B gene in Chinese populations. Meta-analysis showed there to be no correlation between the C-allele and TT genotype and the occurrence of T2DM. However, a subgroup analysis based on the HardyWeinberg equilibrium (HWE) revealed that the frequency of the CC genotype was significantly lower in the T2DM group than in the control group (OR: 0.88; 95\% CI: 0.78, 1.00; $\mathrm{P}=0.049$ ). Seven articles reported the rs10830963 variant of the MTNR1B gene among Chinese populations. Meta-analysis discovered that the G-allele was correlated with the T2DM occurrence. The frequency of the G-allele in the T2DM group was 1.07 times that in the control group (95\% CI: 1.02, 1.12). The GG genotype was associated with the occurrence of T2DM, with its frequency in the T2DM group 1.15 times that in the control group (95\% CI: $1.05,1.25)(\mathrm{P}<0.05)$; however, there was no correlation between the CC genotype and T2DM.

Conclusions: There is a correlation between the CC genotype of the rs1387153 variant in the MTNR1B gene and T2DM in the Chinese population, although this finding needs to be verified in studies with large sample sizes. The G-allele and GG genotype of the rs10830963 variant in the MTNR1B gene are associated with the occurrence of T2DM.

Keywords: Type 2 diabetes mellitus (T2DM); melatonin receptor 1B (MTNR1B); gene polymorphisms; metaanalysis

Submitted Feb 13, 2020. Accepted for publication Apr 24, 2020.

doi: 10.21037/apm-20-691

View this article at: http://dx.doi.org/10.21037/apm-20-691 


\section{Introduction}

Diabetes mellitus, a group of metabolic diseases characterized by hyperglycemia stemming from a variety of causes, is the ninth leading cause of death in the world. By 2040, there will be an estimated 642 million diabetic patients worldwide, $90 \%$ of whom will be diagnosed with type 2 diabetes mellitus (T2DM) (1).

$\mathrm{T} 2 \mathrm{DM}$ is a progressive insulin deficiency, the core factor of which is insulin resistance. Its mechanisms are pathogenically complex, and its occurrence is attributed to a combination of genetic and environmental factors and personal lifestyle habits. The complications brought about by T2DM often affect multiple organs. With high rates of disability and mortality, it places heavy disease and economic burdens on both patients' families and society (2).

Melatonin (MLT) is a neuroendocrine hormone that binds to MLT receptors on islet cells and inhibits insulin secretion (3). Studies have revealed that multiple gene variants of melatonin receptor 1B (MTNR1B) gene are susceptibility genes in T2DM; the single-nucleotide polymorphisms (SNP) of the rs10830963 and rs1387153 variants, for instance, are associated with $\mathrm{T} 2 \mathrm{DM}(4,5)$. However, no correlation has been found between the MTNR1B variants and T2DM $(6,7)$. A meta-analysis by Shen and Jin (8) found that MTNR1B variant rs10830963 was correlated with T2DM, but the results depended on the race of the subjects. Moreover, their analysis did not take Chinese literature into account and thus the relationship between MTNR1B variants and T2DM in the Chinese population could not be reflected. Therefore, in the current study, we explored the relationship between MTNR1B variants and T2DM in Chinese populations, in an attempt to clarify the pathogenesis of T2DM and provide a theoretical basis for the treatment of T2DM.

We present the following article in accordance with the PRISMA reporting checklist (available at http://dx.doi. org/10.21037/apm-20-691).

\section{Methods}

\section{Literature search}

A systematic literature search of English-language databases including PubMed, Embase, and Medline, and Chineselanguage databases including China National Knowledge Infrastructure (CNKI), Wanfang Database, and CQVIP, was conducted to collect relevant articles published up to January 31, 2020. The keywords applied to search the titles and abstracts in the English-language databases included: "melatonin receptor type 1B" or "MTNR1B"; "polymorphism*" AND "variant" or "mutation" or "genotype" or "allele" AND "type 2 diabetes mellitus" or "T2DM". The corresponding Chinese keywords and the same search strategies were used to search the Chineselanguage databases. The references of the returned articles were also searched to ensure all relevant articles were identified.

\section{Inclusion and exclusion criteria}

Articles were included according to the following criteria: (I) all the subjects in the T2DM group were T2DM patients and those in the control group were healthy controls; (II) studies involving MTNR1B gene polymorphisms (at any variant); (III) case-control studies; (IV) the frequencies of each genotype in the T2DM group and the control group were available in the original articles; and $(\mathrm{V})$ the study was carried out on Chinese populations. Articles that met the following criteria were excluded from the meta-analysis: (I) case reports, reviews, summaries, comments, and meeting articles; (II) studies with a Newcastle-Ottawa Scale (NOS) score below 5; (III) inadequate data. For duplicate publications, only the article with the largest sample size was included.

\section{Literature screening and data extraction}

The search results were screened sequentially by title, abstract, and full text using the pre-defined inclusion/ exclusion criteria by two independent researchers. Data extraction and quality assessment were performed using a standardized form. The basic data extracted from the literature included: name of the first author; year of publication; language of the publication; sample sizes of the T2DM and control groups; and the frequency of each variant of the MTNR1B gene. When substantial differences existed between the two reviewers, a third reviewer was appointed to resolve the dispute.

\section{Quality evaluation of the articles}

The quality of the literature was assessed according to the NOS (9), taking into account the selection of the T2DM and control groups, comparability of the study, and exposure/outcome. The NOS ranges from to nine stars, and a higher score represents higher quality of a specific study. 


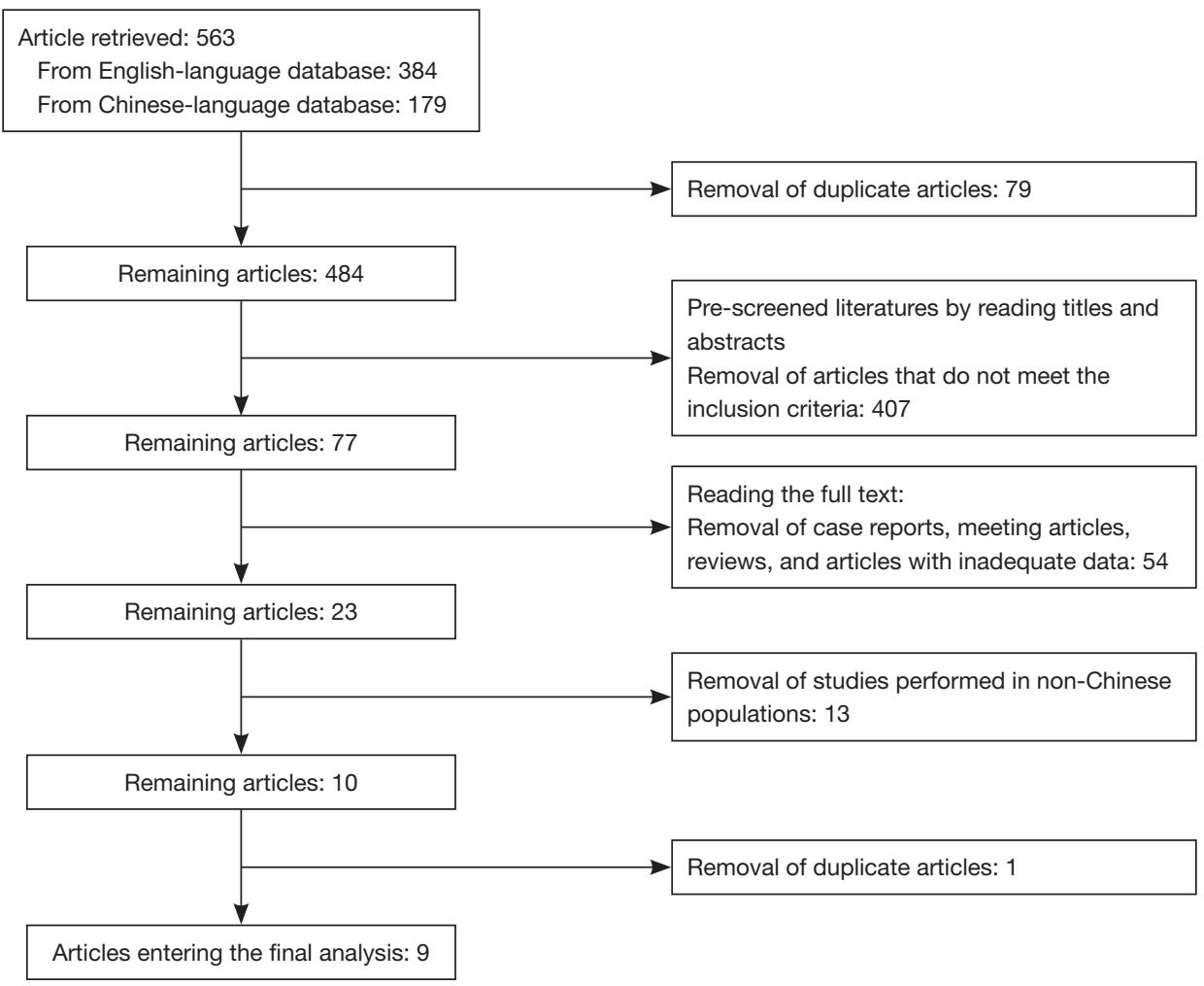

Figure 1 Flowchart of references retrieval.

\section{Statistical analysis}

The Hardy-Weinberg equilibrium (HWE) was checked first. The correlation between the MTNR1B gene polymorphisms and T2DM was pooled with the odds ratio $(\mathrm{OR})$ and $95 \%$ confidence interval $(\mathrm{CI})$. A $\mathrm{P}$ value of $\leq 0.05$ indicated statistical significance. Before the data were pooled, the $\mathrm{I}^{2}$ statistic was used to quantify the heterogeneity. When $\mathrm{I}^{2}$ was $\leq 50 \%$, a fixed effect model was used. For studies with an $\mathrm{I}^{2}$ of $>50 \%$, subgroup analysis and sensitivity analysis were performed to check HWE; if the source of the heterogeneity could not be identified, a random effects model was used for meta-analysis. The presence of publication bias was detected using a funnel plot and assessed with Begg's rank correlation and Egger's weighted regression tests. All of the statistical analyses were performed by using the "meta" package of the R3.5.1 software.

\section{Results}

\section{Included articles}

A total of 564 Chinese- and English-language articles were retrieved through the literature searches. After screening, 9 articles (4-7,10-14) met the inclusion criteria; the literature searching and screening flowchart is shown in Figure 1. These articles involved 10,127 subjects (T2DM patients) in the T2DM group and 10,885 subjects (healthy individuals) in the control group. Of the nine articles four were published in Chinese and five in English; two MTNR1B variants, rs1387153 and rs10830963, were described; two articles $(5,6)$ considered both variants, whereas the remaining seven articles focused on one variant. Seven articles conformed to HWE $(\mathrm{P} \geq 0.05)$. The quality of the included articles was high, with NOS scores ranging from 7 to 8 points. The characteristics of the included literature are shown in Table 1.

\section{Relationship between rs1387153 SNPs and T2DM}

Four studies (3,551 T2DM cases and 3,709 controls) investigated the relationship between rs1387153 SNPs and T2DM. One of these articles did not conform to HWE.

\section{Analysis of alleles (C vs. T)}

Meta-analysis of the $\mathrm{C}$-allele revealed high allelic heterogeneity 
Table 1 Basic characteristics of the included articles on the relationship between variants of MTNR1B and T2DM in Chinese populations

\begin{tabular}{|c|c|c|c|c|c|c|c|c|c|c|c|c|c|}
\hline Authors/year & Language & Variant & \multicolumn{4}{|c|}{ T2DM group } & \multicolumn{4}{|c|}{ Control group } & $\begin{array}{l}\text { HWE test } \\
\chi^{2} \text { value }\end{array}$ & $\begin{array}{l}\text { HWE test } \\
P \text { value }\end{array}$ & $\begin{array}{l}\text { NOS } \\
\text { score }\end{array}$ \\
\hline Liu 2011 (10) & Chinese & rs1387153 & 105 & 150 & 40 & 295 & 84 & 118 & 37 & 239 & 0.176 & 0.674 & 7 \\
\hline Liang 2010 (5) & Chinese & rs1387153 & 23 & 51 & 17 & 91 & 27 & 49 & 13 & 89 & 1.482 & 0.223 & 7 \\
\hline Li 2012 (4) & Chinese & rs1387153 & 234 & 442 & 577 & 1253 & 720 & 90 & 530 & 1340 & 99.795 & $<0.001$ & 8 \\
\hline Rönn 2009 (11) & English & rs10830963 & 371 & 553 & 241 & 1165 & 374 & 558 & 173 & 1105 & 2.189 & 0.086 & 7 \\
\hline Liang 2010 (5) & Chinese & rs10830963 & 15 & 47 & 29 & 91 & 25 & 46 & 18 & 89 & 0.143 & 0.086 & 7 \\
\hline Kan 2010 (6) & English & rs10830963 & 585 & 960 & 367 & 1912 & 675 & 989 & 350 & 2014 & 0.142 & 0.707 & 7 \\
\hline Tam 2010 (12) & English & rs10830963 & 448 & 633 & 261 & 1342 & 523 & 789 & 332 & 1644 & 1.2 & 0.274 & 8 \\
\hline Gao 2016 (7) & English & rs10830963 & 243 & 347 & 134 & 724 & 280 & 350 & 129 & 759 & 1.198 & 0.274 & 7 \\
\hline
\end{tabular}

*, the genotypes of variant rs10830963 were CC, CG, and GG. MTNR1B, melatonin receptor 1B; T2DM, type 2 diabetes mellitus; HWE, Hardy-Weinberg equilibrium; NOS, Newcastle-Ottawa Scale.

$\left(\mathrm{I}^{2}=98 \%\right)$. OR was estimated using a random effects model, which showed there to be no significant correlation between the C-allele and risk of T2DM; the frequency of the C-allele in the T2DM group was 0.76 times that in the control group (95\% CI: $0.46,1.24)$. The allelic heterogeneity was significantly reduced to $0 \%$ in the subgroups that conformed to HWE. Estimates based on a fixed effects model showed that the $\mathrm{C}$-allele had no statistical correlation with the occurrence of T2DM (OR $=0.93,95 \%$ CI: 0.86, 1.01) (Figure 2).

\section{Analysis of genotypes (CC vs. CT + TT)}

Meta-analysis of the CC genotype revealed high heterogeneity $\left(\mathrm{I}^{2}=98 \%\right)$. OR was estimated using a random effects model, which showed there to be no significant correlation between the CC genotype and risk of T2DM; the frequency of the CC genotype in the T2DM group was 0.60 times that in the control group (95\% CI: $0.24,1.53)$. The heterogeneity was significantly reduced to $0 \%$ in the subgroups that conformed to HWE. Estimates based on a fixed effects model showed that the CC genotype was significantly correlated with the occurrence of T2DM (OR $=0.88,95 \%$ CI: 0.78, 1.00) (Figure 3).

\section{Analysis of genotypes (TT vs. CT+CC)}

Meta-analysis of the TT genotype revealed low heterogeneity
$\left(\mathrm{I}^{2}=34 \%\right)$. OR was estimated using a fixed effects model, which showed significant correlation between the TT genotype and risk of T2DM; the frequency of the TT genotype in the T2DM group was 1.17 times that in the control group (95\% CI: 1.05, 1.31). The heterogeneity was significantly reduced to $0 \%$ in the subgroups that conformed to HWE. Estimates based on a fixed effects model showed that the CC genotype had no statistical correlation with the occurrence of T2DM (OR $=1.06,95 \%$ CI: 0.91, 1.24) (Figure 4).

\section{Relationship between rs10830963 SNPs and T2DM}

Seven studies (3,551 T2DM cases and 3,709 controls) investigated the relationship between rs10830963 SNPs and T2DM.

\section{Meta-analysis of alleles (G vs. C)}

Meta-analysis of the G-allele revealed low allelic heterogeneity $\left(\mathrm{I}^{2}=50 \%\right)$. OR was estimated using a fixed effects model, which showed significant correlation between the $G$ allele and risk of T2DM; the frequency of the G allele in the T2DM group was 1.07 times that in the control group (95\% CI: 1.02, 1.12). The heterogeneity was significantly increased to $54 \%$ in the subgroups that conformed to HWE. Estimates based on a random effects model showed 


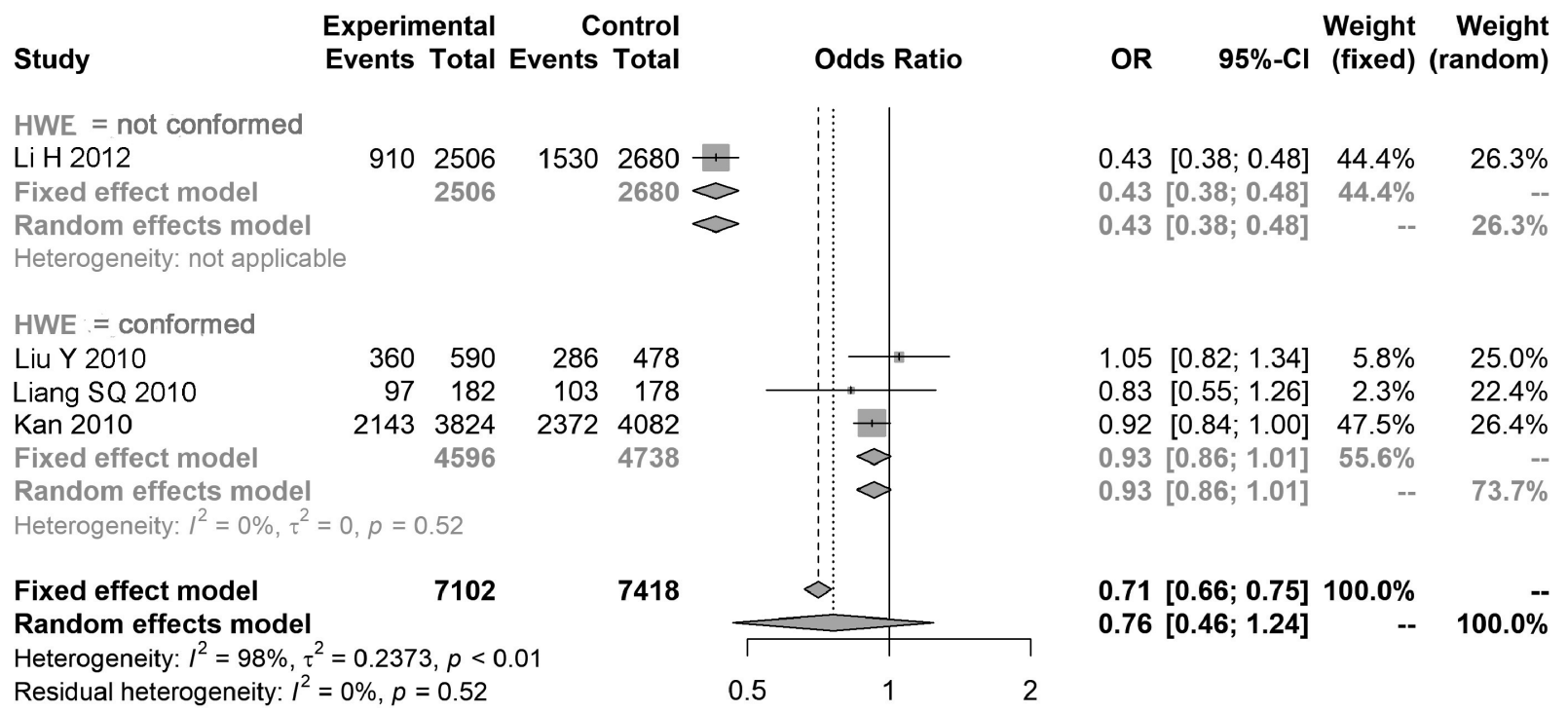

Figure 2 Meta-analysis of the correlation of the C-allele in rs1387153 in the MTNR1B gene with T2DM in Chinese populations. MTNR1B, melatonin receptor 1B; T2DM, type 2 diabetes mellitus.

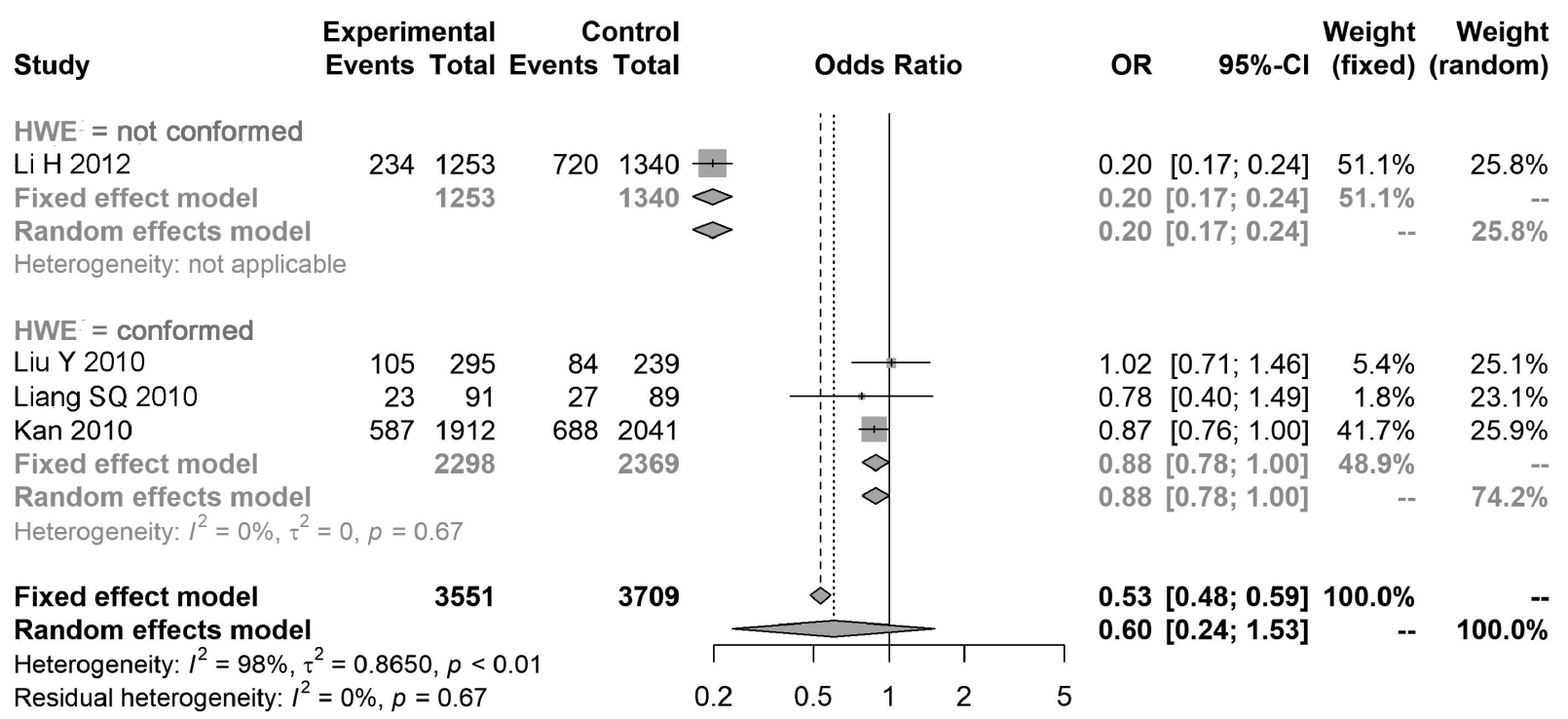

Figure 3 Meta-analysis of the correlation of the CC genotype in rs1387153 in the MTNR1B gene with T2DM in Chinese populations. MTNR1B, melatonin receptor 1B; T2DM, type 2 diabetes mellitus.

that the $G$ allele had no significant correlation with the occurrence of T2DM (OR $=1.10 ; 95 \%$ CI: $1.01,1.19)$ (Figure 5).

\section{Analysis of genotypes (CC vs. CG + GG)}

Meta-analysis of the CC genotype revealed low heterogeneity $\left(\mathrm{I}^{2}=36 \%\right)$. OR was estimated using a fixed effects model, which showed no significant correlation between the $\mathrm{CC}$ genotype and risk of T2DM; the frequency of the CC genotype in the T2DM group was 0.95 times that in the control group (95\% CI: $0.88,1.02)$. The heterogeneity was slightly reduced to $34 \%$ in the subgroups that conformed to HWE. Estimates based on a fixed effects model showed that the CC genotype had no statistical correlation with 


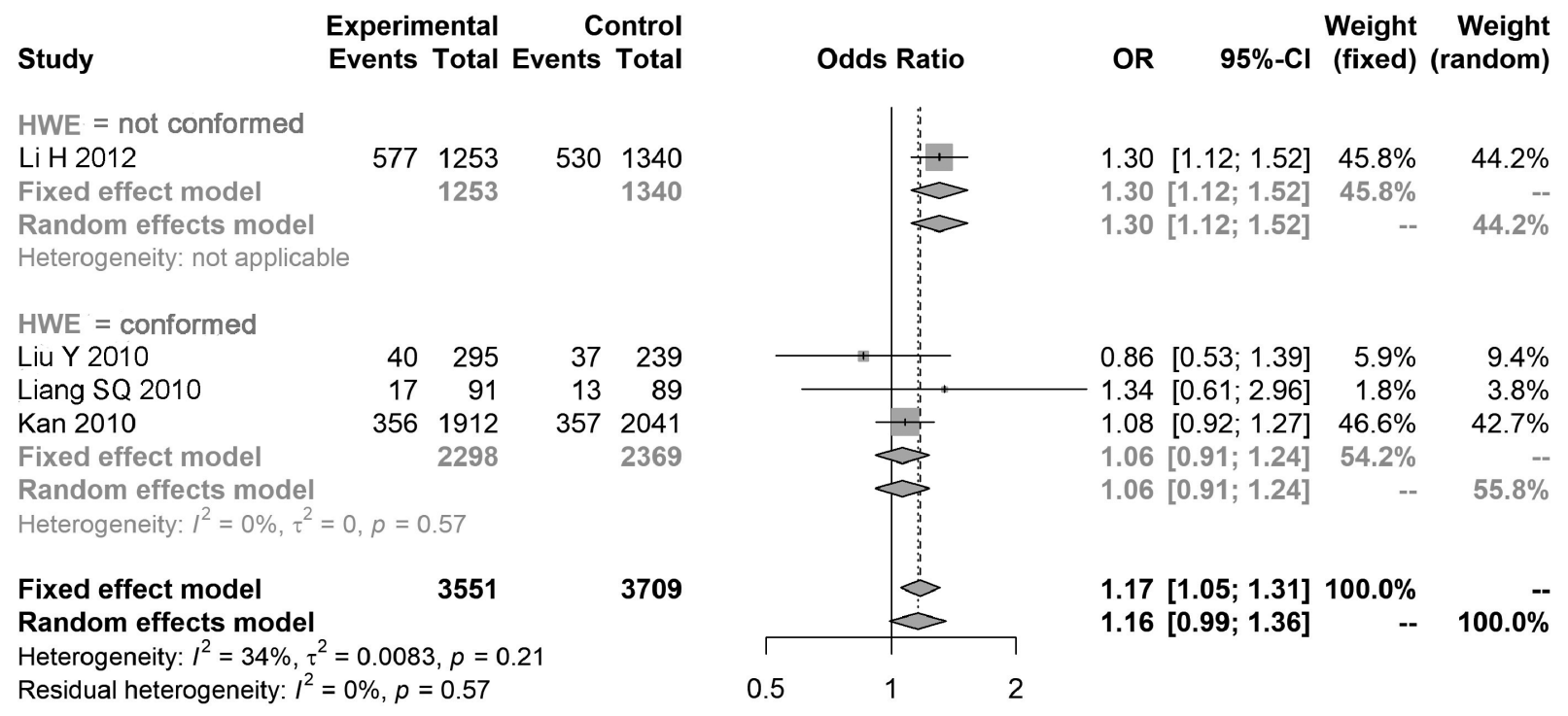

Figure 4 Meta-analysis of the correlation of the TT genotype in rs1387153 in the MTNR1B gene with T2DM in Chinese populations. MTNR1B, melatonin receptor 1B; T2DM, type 2 diabetes mellitus

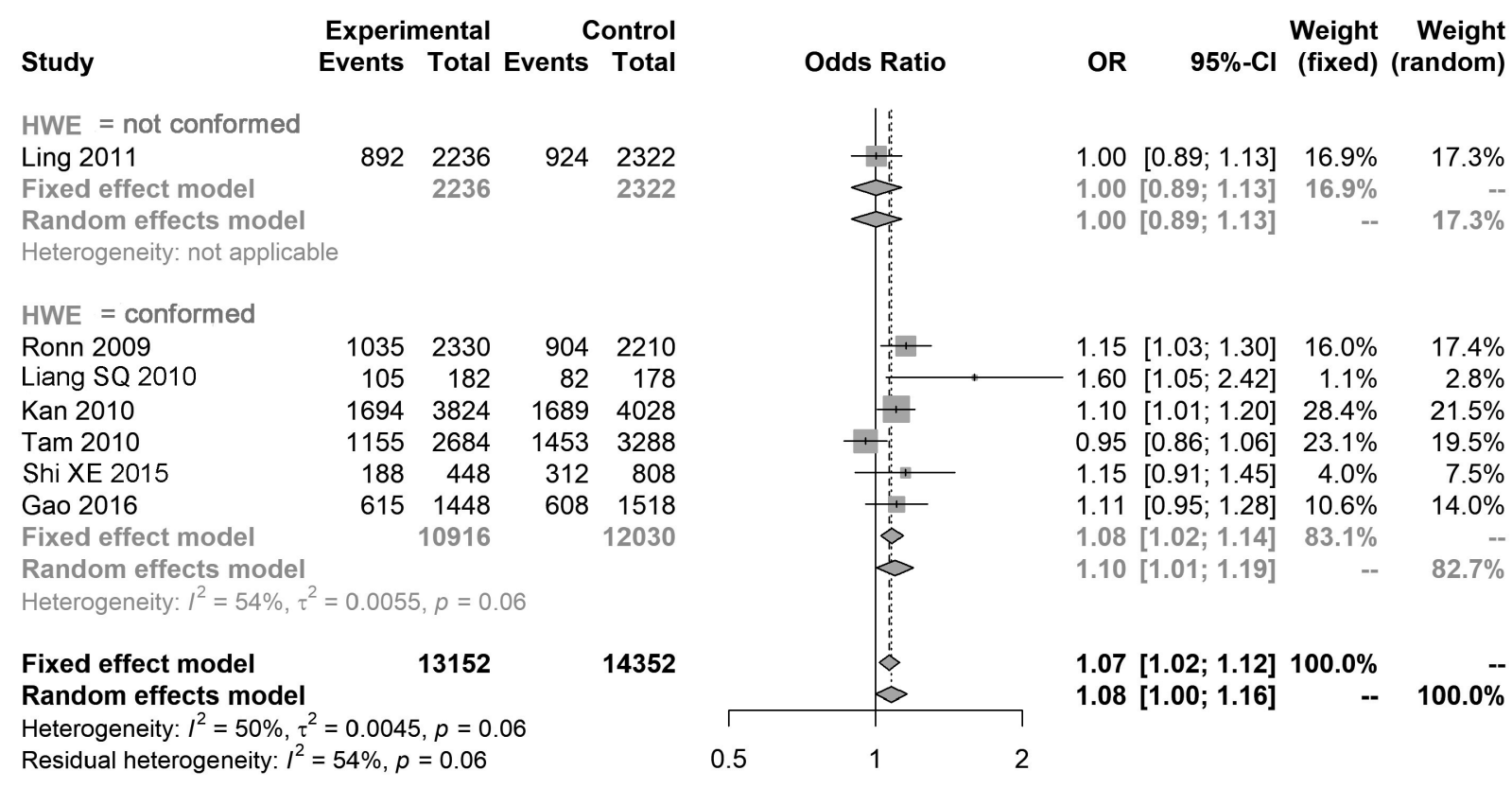

Figure 5 Meta-analysis of the correlation of the G allele in rs10830963 in the MTNR1B gene with T2DM in Chinese populations. MTNR1B, melatonin receptor 1B; T2DM, type 2 diabetes mellitus.

the occurrence of T2DM (OR $=0.93,95 \%$ CI: $0.86,1.01)$

(Figure 6).

Meta-analysis of genotypes (GG vs. CG + CC)

Meta-analysis of the GG genotype revealed low heterogeneity
$\left(\mathrm{I}^{2}=50 \%\right)$. OR was estimated using a fixed effect model, which showed there was significant correlation between the GG genotype and risk of T2DM; the frequency of the GG genotype in the T2DM group was 1.15 times that in the control group (95\% CI: 1.05, 1.25). The heterogeneity 


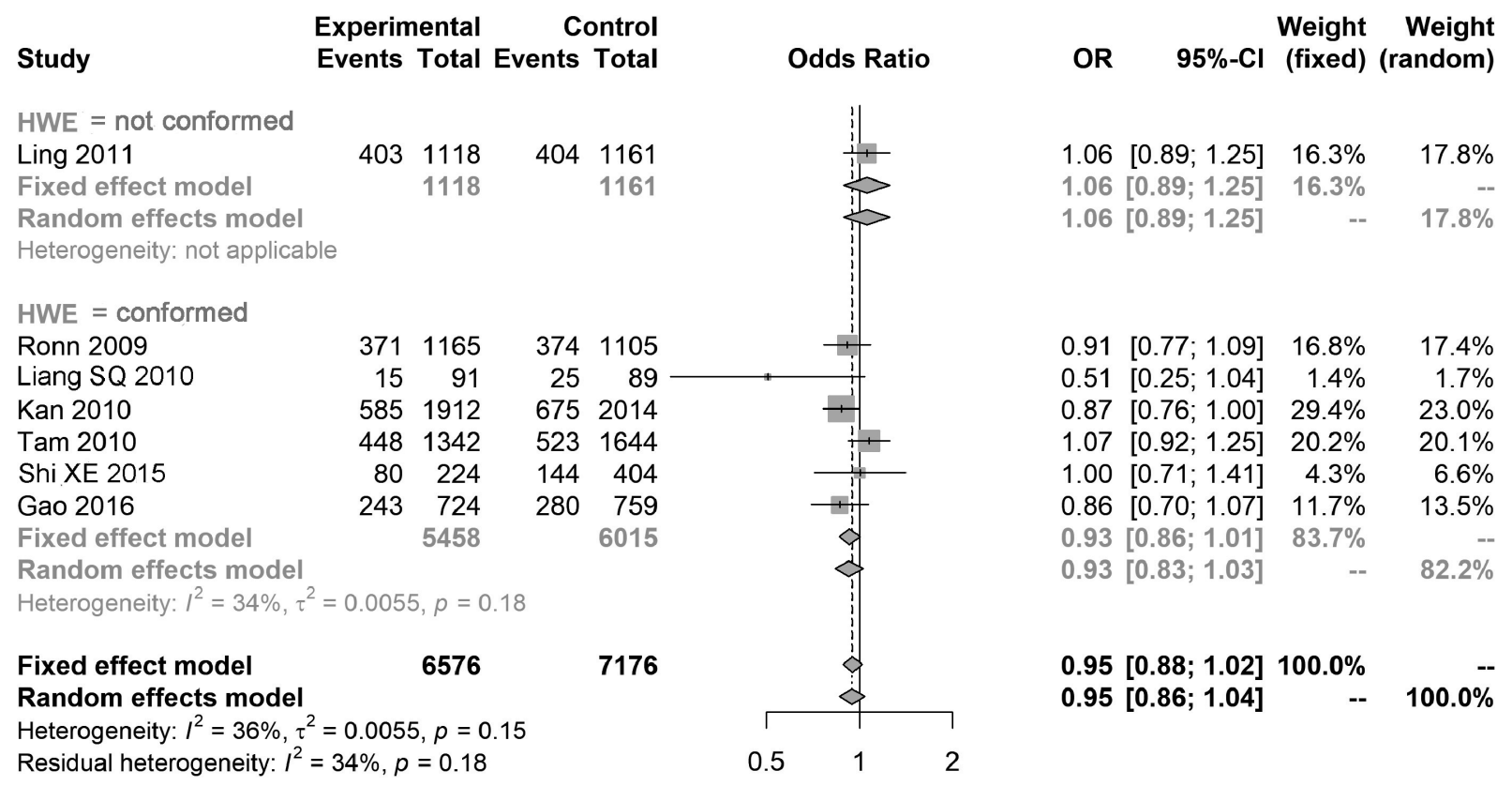

Figure 6 Meta-analysis of the correlation of the CC genotype in rs10830963 in the MTNR1B gene with T2DM in Chinese populations. MTNR1B, melatonin receptor 1B; T2DM, type 2 diabetes mellitus.

was significantly increased to $58 \%$ in the subgroups that conformed to HWE. Estimates based on a random effects model showed the GG genotype was significantly correlated with the occurrence of T2DM (OR $=1.20 ; 95 \% \mathrm{CI}: 1.02$, 1.42) (Figure 7).

\section{Publication bias and sensitivity}

Due to the small number $(<10)$ of articles included in this meta-analysis, the statistical power was low and the funnel chart was not sufficient to identify any publication bias. Therefore, this meta-analysis did not assess publication bias.

Sensitivity analysis was performed after one-by-one removal of the articles, and the results did not change significantly.

\section{Discussion}

In our current analysis, the C-allele and TT genotype of the rs1387153 variant of the MTNR1B gene were shown to have no correlation with the occurrence of T2DM among Chinese populations. However, subgroup analysis based on the HWE revealed that the frequency of the CC genotype was significantly lower in the T2DM group than in the control group (OR: 0.88; 95\% CI: 0.78, 1.00) $(\mathrm{P}=0.049)$. In addition, no significant correlation was found between the TT genotype and the risk of T2DM. The G-allele and GG genotype of the rs10830963 variant in the MTNR1B gene were associated with T2DM occurrence, as the frequency of the G-allele in the T2DM group was 1.07 times that in the control group (95\% CI: 1.02, 1.12). An association was revealed between the GG genotype and the occurrence of T2DM, with its frequency in the T2DM group 1.15 times that in the control group (95\% CI: 1.05, 1.25) $(\mathrm{P}<0.05)$; however, the CC genotype and T2DM were not found to be correlated.

The MTNR1B gene is located on human chromosome 11q21-q22 and comprises two exons and one intron. With a total mRNA length of 1,662 bp, it encodes a total of 362 amino acids. Its translation product is MLT receptor 2 (MT2), which binds to MLT and subsequently mediates the inhibition of insulin secretion (3). The secretion of MLT and insulin is regulated by the circadian rhythm. Circadian rhythm disorders can lead to the occurrence of metabolic diseases, including diabetes (15). Studies have discovered that MT2 is a G protein-coupled receptor which is highly expressed in the islet tissue of T2DM patients. Its binding to MLT affects the $\beta$-cell cAMP level in human islets, thereby reducing insulin secretion and increasing the risk of T2DM $(16,17)$. To date, studies have found that SNPs 


\begin{tabular}{|c|c|c|c|c|}
\hline Study & \multicolumn{2}{|c|}{ Experimental } & \multicolumn{2}{|c|}{$\begin{array}{r}\text { Control } \\
\text { ents Total }\end{array}$} \\
\hline $\begin{array}{l}\text { HWE = not conformed } \\
\text { Ling } 2011 \\
\text { Fixed effect model } \\
\text { Random effects model } \\
\text { Heterogeneity: not applica }\end{array}$ & 177 & $\begin{array}{l}1118 \\
1118\end{array}$ & 167 & $\begin{array}{l}1161 \\
1161\end{array}$ \\
\hline HWE = conformed & & & & \\
\hline $\begin{array}{l}\text { Ronn } 2009 \\
\text { Liang SQ } 2010\end{array}$ & $\begin{array}{r}241 \\
29\end{array}$ & $\begin{array}{r}1165 \\
91\end{array}$ & $\begin{array}{r}173 \\
18\end{array}$ & $\begin{array}{r}1105 \\
89\end{array}$ \\
\hline Kan 2010 & 367 & 1912 & 350 & 2014 \\
\hline Tam 2010 & 261 & 1342 & 332 & 1644 \\
\hline Shi XE 2015 & 44 & 224 & 52 & 404 \\
\hline Gao 2016 & 134 & 724 & 129 & 759 \\
\hline $\begin{array}{l}\text { Fixed effect model } \\
\text { Random effects model } \\
\text { Heterogeneity: } I^{2}=58 \% \text {, }\end{array}$ & $2=0.0217$ & $\begin{array}{l}5458 \\
7, p=0\end{array}$ & & 6015 \\
\hline $\begin{array}{l}\text { Fixed effect model } \\
\text { Random effects model } \\
\text { Heterogeneity: } I^{2}=50 \%, \tau \\
\text { Residual heterogeneity: } I^{2}\end{array}$ & $\begin{array}{l}=0.015 \\
=58 \%, p\end{array}$ & $\begin{array}{l}1, p= \\
=0.03\end{array}$ & & 7176 \\
\hline
\end{tabular}

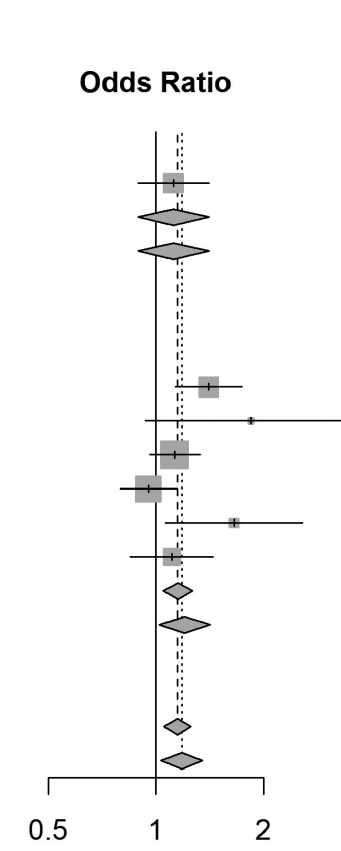

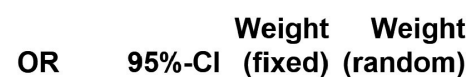

$\begin{array}{rrr}1.12[0.89 ; 1.41] & 14.7 \% & 16.4 \% \\ 1.12[0.89 ; 1.41] & 14.7 \% & -- \\ 1.12[0.89 ; 1.41] & -- & 16.4 \%\end{array}$

$1.41[1.13 ; 1.74] \quad 15.0 \% \quad 17.4 \%$

$1.84[0.94 ; 3.64] \quad 1.3 \% \quad 3.5 \%$

$1.13[0.96 ; 1.33] \quad 29.3 \% \quad 21.5 \%$

$0.95[0.80 ; 1.14] \quad 25.6 \% \quad 20.0 \%$

$1.65[1.07 ; 2.57] \quad 3.2 \% \quad 7.2 \%$

$1.11[0.85 ; 1.45] \quad 10.9 \% \quad 14.1 \%$

$1.15[1.05 ; 1.27] \quad 85.3 \%$

$1.20[1.02 ; 1.42] \quad--\quad 83.6 \%$

$\begin{array}{lrr}1.15 & {[1.05 ; 1.25]} & 100.0 \% \\ 1.18[1.03 ; 1.35] & -- & 100.0 \%\end{array}$

Figure 7 Meta-analysis of the correlation of the TT genotype in rs10830963 in the MTNR1B gene with T2DM in Chinese populations. MTNR1B, melatonin receptor 1B; T2DM, type 2 diabetes mellitus.

of the rs1387153 and rs10830963 variants of the MTNR1B gene are associated with the pathogenesis of T2DM, although the results of these studies are inconsistent.

The rs138715 variant in the MTNR1B gene is located on chromosome 11, $28.3 \mathrm{~kb}$ upstream of the gene. Its SNPs include homozygous CC, heterozygous CT, and wild-type TT. In our current analysis, the C-allele and CC genotype of the rs1387153 variant in the MTNR1B gene were not revealed to be associated with the occurrence of T2DM. Notably, the heterogeneity of the four relevant articles was as high as $98 \%$. Subgroup analysis based on HWE revealed a significant reduction in heterogeneity. Meta-analysis based on HWE found that the CC genotype of the rs 1387153 variant in the MTNR1B gene is a protective factor of T2DM, and no significant change was shown in the sensitivity analysis. However, since the number of relevant articles was small, this finding needs to be further verified in studies with larger sample sizes comprising Chinese populations.

The rs 10830963 variant is located in the intron of the MTNR1B gene. With reduced beta cell function or increased fasting blood glucose levels, A large study carried out in a European population revealed that the SNP of the rs10830963 variant increases the risk of T2DM (16). In our current analysis, the G-allele of the rs 10830963 variant in the MTNR1B gene was associated with the occurrence of T2DM; in addition, the GG genotype was associated with the occurrence of T2DM, with a frequency significantly higher in the T2DM group than in the control group. Subgroup analysis based on HWE did not identify any decrease in the heterogeneity, indicating that the heterogeneity in this meta-analysis cannot be attributed to disequilibrium among the HWE populations. Further literature analysis showed that most of the studies were performed in Han Chinese populations, whereas the study carried out by Shi et al. (14) targeted the Bao'an ethnic group in Jishishan, Gansu Province, which may have caused heterogeneity. Furthermore, differences in age, gender, and T2DM history among the included studies also contributed to the heterogeneity observed in this meta-analysis.

A meta-analysis performed by Shen and Jin (8) found that no correlation existed between the SNPs of the rs1387153 variant in the MTNR1B gene and T2DM; however, the distribution of the $\mathrm{C}$ and $\mathrm{G}$ alleles of rs10830963 differed significantly, and the frequency of the C-allele in the T2DM group was 0.97 times that in the control group $(95 \% \mathrm{CI}$ : $0.95,1.00)$. Their conclusions on the relationship between the SNPs of the rs1387153 variant in the MTNR1B gene and T2DM are inconsistent with the results of our current meta-analysis, mainly due to the difference in the 
included articles, thus further proving that the distribution of polymorphisms in the MTNR1B gene is race-specific. Therefore, it is of great significance that SNP analysis of different races and ethnic groups is conducted. However, due to the lack of data on the SNPs of the rs 1387153 variant in the MTNR1B gene, population-based studies with large sample sizes are needed.

Our meta-analysis had the following limitations: (I) high heterogeneity existed between the studies on the SNPs of the rs1387153 variant of the MTNR1B gene. In addition to the differences in the population characteristics, the reagents and equipment used in different studies might also have contributed to the high heterogeneity. However, stratified analysis is still not possible due to limited data for relevant populations. (II) The research results might have been influenced by gene-gene and/or gene-environment interactions; however, these were not described in the included articles. (III) In this analysis, the CC genotype of the rs1387153 variant in the MTNR1B gene was investigated in studies with small sample sizes, and the relevant conclusions need to be further verified.

Since the quality of the case-control studies involving Chinese populations included in this analysis was high, the following conclusions can be drawn: (I) the $\mathrm{C}$ allele and TT genotypes of rs1387153 in the MTNR1B gene are not associated with the risk of T2DM in Chinese populations, whereas the CC genotype of the rs1387153 variant in the MTNR1B gene is weakly correlated with T2DM. However, these findings need to be further verified in studies with large sample sizes. The G-allele and GG genotype of the rs10830963 variant in the MTNR1B gene are associated with the occurrence of T2DM.

\section{Acknowledgments}

Funding: None.

\section{Footnote}

Reporting Checklist: The authors have completed the PRISMA reporting checklist. Available at http://dx.doi. org/10.21037/apm-20-691

Conflicts of Interest: All authors have completed the ICMJE uniform disclosure form (available at http://dx.doi. org/10.21037/apm-20-691). The authors have no conflicts of interest to declare.
Ethical Statement: The authors are accountable for all aspects of the work in ensuring that questions related to the accuracy or integrity of any part of the work are appropriately investigated and resolved.

Open Access Statement: This is an Open Access article distributed in accordance with the Creative Commons Attribution-NonCommercial-NoDerivs 4.0 International License (CC BY-NC-ND 4.0), which permits the noncommercial replication and distribution of the article with the strict proviso that no changes or edits are made and the original work is properly cited (including links to both the formal publication through the relevant DOI and the license). See: https://creativecommons.org/licenses/by-nc-nd/4.0/.

\section{References}

1. Zheng Y, Ley SH, Hu FB. Global aetiology and epidemiology of type 2 diabetes mellitus and its complications. Nat Rev Endocrinol 2018;14:88-98.

2. Abdul-Ghani M, Jayyous A, Asaad N, et al. Pioglitazone and cardiovascular risk in T2DM patients: is it good for all? Ann Transl Med 2018;6:192.

3. Stumpf I, Mühlbauer E, Peschke E. Involvement of the cGMP pathway in mediating the insulin-inhibitory effect of melatonin in pancreatic beta-cells. J Pineal Res 2008;45:318-27.

4. Li H. Correlations of mutation sites of gene MTNR1B rs1387153 and gene KLT14 rs972283 with T2DM in a northern China population. Dalian Medical University, 2012.

5. Liang S. Correlation between MTNR1B gene and type 2 diabetes in Han Chinese people in Jilin area. Jilin University, 2010.

6. Kan MY, Zhou DZ, Zhang D, et al. Two susceptible diabetogenic variants near/in MTNR1B are associated with fasting plasma glucose in a Han Chinese cohort. Diabet Med 2010;27:598-602.

7. Gao K, Wang J, Li L, et al. Polymorphisms in Four Genes (KCNQ1 rs151290, KLF14 rs972283, GCKR rs780094 and MTNR1B rs10830963) and Their Correlation with Type 2 Diabetes Mellitus in Han Chinese in Henan Province, China. Int J Environ Res Public Health 2016. doi: 10.3390/ijerph13030260.

8. Shen LL, Jin Y. Effects of MTNR1B genetic variants on the risk of type 2 diabetes mellitus: A meta-analysis. Mol Genet Genomic Med 2019;7:e611.

9. Wells GA, Shea B, O'Connell D, et al. The Newcastle- 
Ottawa Scale (NOS) for assessing the quality of non randomised studies in meta-analyses. Available online: http://www.ohri.ca/programs/clinical_epidemiology/ oxford.asp. 2016.

10. Liu Y. Correlation between MTNR1B gene polymorphisms and type 2 diabetes in Han populations in Ningxia. Ningxia Medical University, 2011.

11. Rönn T, Wen J, Yang Z, et al. A common variant in MTNR1B, encoding melatonin receptor $1 \mathrm{~B}$, is associated with type 2 diabetes and fasting plasma glucose in Han Chinese individuals. Diabetologia 2009;52:830-3.

12. Tam CH, Ho JS, Wang Y, et al. Common polymorphisms in MTNR1B, G6PC2 and GCK are associated with increased fasting plasma glucose and impaired beta-cell function in Chinese subjects. PLoS One 2010;5:e11428.

13. Ling Y, Li X, Gu Q, Chen H, Lu D, Gao X. A common polymorphism rs3781637 in MTNR1B is associated

Cite this article as: Zhang X, Xie L, Zhong M, Yang B, Yang Q, Yang H, Xie C. The association between melatonin receptor 1B gene polymorphisms and type 2 diabetes mellitus (T2DM) in Chinese populations: a meta-analysis. Ann Palliat Med 2020;9(3):957-966. doi: 10.21037/apm-20-691 with type 2 diabetes and lipids levels in Han Chinese individuals. Cardiovasc Diabetol 2011;10:27.

14. Shi X, Peng X, Wang Z, et al. Correlation between MTNR1B gene rs10830963 polymorphism and type 2 diabetes mellitus in Gansu Bao-an population. Clinical Focus 2015;30:544-7.

15. Peschke E, Stumpf I, Bazwinsky I, et al. Melatonin and type 2 diabetes-a possible link? J Pineal Res 2007;42:350-8.

16. Prokopenko I, Langenberg C, Florez JC, et al. Variants in MTNR1B influence fasting glucose levels. Nat Genet 2009;41:77-81.

17. Lyssenko V, Nagorny CL, Erdos MR, et al. Common variant in MTNR1B associated with increased risk of type 2 diabetes and impaired early insulin secretion. Nat Genet 2009;41:82-8. 